\title{
Italian society of colorectal surgery recommendations for good clinical practice in colorectal surgery during the novel coronavirus pandemic
}

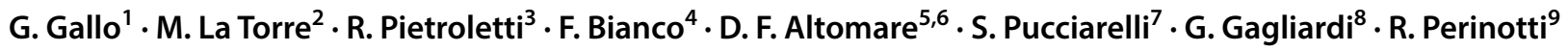

Received: 2 April 2020 / Accepted: 3 April 2020 / Published online: 14 April 2020

(c) Springer Nature Switzerland AG 2020

The first cases of severe acute respiratory syndrome coronavirus 2 (SARS-COV-2) infection, the so-called 2019 novel COronaVIrus Disease (COVID-19), were first reported in Wuhan, Hubei Province, China in December 2019 [1].

After 3 months and about 125,000 cases in more than 118 countries, on the 12 th of March, the WHO defined the spread of SARS-COV-2 as a pandemic [2].

Since the first case of SARS-CoV-2 was confirmed in Italy on the 21st of February, the northern regions first [3] (because first shocked by the event) and then the southern ones, to avoid further spread of infection, have closed schools, universities, museums, and all other public places, also canceling all the events that could create crowds such as football matches or musical concerts.

In this chaotic moment, the consequences of this severe and indispensable quarantine have been overwhelming, especially on the Public Health System (Sistema Sanitario

G. Gallo

gaetanogallo1988@gmail.com

1 Department of Medical and Surgical Sciences, University of Catanzaro, Catanzaro, Italy

2 Coloproctology Unit, S. Anna Clinic, Pomezia, Italy

3 Coloproctology Unit, Hospital Val Vibrata, University of L'Aquila, L'Aquila, Italy

4 General Surgery Unit, S. Leonardo Hospital, Castellammare Di Stabia, Napoli, Italy

5 Functional and Oncologic Colorectal Unit, Department of Emergency and Organ Transplantation, University of Bari, Bari, Italy

6 IRCCS Ospedale Oncologico Giovanni Paolo II, Bari, Italy

7 Department of Surgery, Oncology and Gastroenterology, University of Padova, Padova, Italy

8 Department of Surgery, University of Illinois At Chicago, Chicago, IL, USA

9 Colorectal Surgical Unit, Department of Surgery, Infermi Hospital, Biella, Italy
Nazionale, SSN), with relevant differences from region to region.

Concerning the field of surgery, elective procedures, including day case surgeries, have been canceled in favor of emergencies. Furthermore, many regions are trying to identify centers of reference where oncological cases that are COVID-19 negative should converge.

During these hard times, there was no possibility to define evidence-based clinical practice guidelines even if several national and international scientific societies are trying to develop recommendations based on common sense [4-6].

However, recommendations are prone to be modified during the evolution of the disease, and it is necessary to review the literature and reassess each clinical case on a daily basis to avoid any delay in diagnosis and treatment of colorectal diseases.

Our recommendations are tailored and based on the impact of the disease on the regional health organizations and according to the emergency level of every single hospital (Table 1). According to these stratifications, the possibility of transferring a patient from a COVID-19 emergency hospital to a low emergency one, to guarantee the same high standard of surgical care, should be considered.

Proctology is one of the subspecialties that has suffered the worst consequences related to COVID-19. In fact, all the outpatient clinics and elective procedures have been postponed with the exception of anorectal emergencies. In these cases, the use of local anesthesia and/or sedation in an outpatient setting can be proposed to avoid hospital admissions.

With regard to other proctological diseases, including functional disorders, a virtual visit, the so-called "telemedicine", must probably be considered as a first-line solution, since it is safe and effective (in this emergency context) and provides a rapid access to specialists who are not immediately available due to the viral outbreak. Furthermore, patients could be temporarily aided through telephone calls, e-mail, or social media. 
Table 1 Priority level and activities

\begin{tabular}{lll}
\hline Priority level & Resources & Activities \\
\hline $\begin{array}{l}\text { High } \\
\text { Moderate }\end{array}$ & $\begin{array}{l}\text { Critical unavailability of hospital resources } \\
\text { Hospital resources close to exhaustion }\end{array}$ & $\begin{array}{l}\text { All elective surgical and endoscopic cases } \\
\text { should be postponed. Surgical care should be } \\
\text { limited to those patients with life-threatening } \\
\text { conditions (lower gastrointestinal bleeding, } \\
\text { perforation, and obstruction), advanced symp- } \\
\text { tomatic tumors or anorectal emergencies }\end{array}$ \\
Low & $\begin{array}{l}\text { Elective oncological colorectal surgery proce- } \\
\text { dures should be performed in COVID-19-neg- } \\
\text { ative settings. Surgery for benign disease } \\
\text { should be postponed until after the peak of the } \\
\text { pandemic is seen }\end{array}$ \\
& &
\end{tabular}

Screening and surveillance endoscopy and all the other diagnostic procedures such as anoscopy, endoanal ultrasound, and anorectal manometry should be postponed according to the recent evidence of fecal COVID-19 transmission and the persistence of the virus in fecal samples for a longer period than in nasopharyngeal swabs [7-10]. Repici et al. [11] have suggested screening all patients by phone the day before an endoscopic procedure, by asking for the presence of symptoms in the last 14 days, a history of contact with COVID-19 positive patients, and a travel history. The patient's temperature should be checked before the procedure and the patient should wear a surgical mask. Upper or lower endoscopy in intermediate- or high-risk patients should be performed wearing special protective equipment and in negative pressure rooms.

Whilst patients with inflammatory bowel disease (IBD) taking immunosuppressive drugs may be at higher risk of infection, the 2nd interview COVID-19 ECCO Taskforce [12] has highlighted that there are no data demonstrating that immunosuppressive therapies increase the risk of complicated COVID-19.

Patients with IBD who are suspected to have a COVID19 infection, if they are not at high risk of flare-up, should stop thiopurines, delay methotrexate injections and delay biologics, and in particular, JAK inhibitors which decrease the number of lymphocytes. Given the short course of the disease (3-4 weeks), these precautions are unlikely to cause flare ups. Steroids have been used to control cytokine release in COVID-19 patients; however, the risks and benefits of steroid treatment in COVID-19-positive IBD patients should be carefully weighed. Physicians are invited to register their COVID-19-positive IBD patients on the SECURE-IBD (Surveillance Epidemiology of Coronavirus Under Research Exclusion) website. As of the 23rd of March, a total of 41 patients from 13 different countries were already enrolled [13].

The management of acute complicated diverticulitis depends on the clinical manifestations and has not undergone changes following the outbreak of COVID-19. An initial conservative approach with observation and antibiotic treatment is recommended. Meanwhile, in COVID-19 positive patients, open surgery may be preferred to laparoscopic surgery for Hinchey 3 and 4 patients to avoid aerosolized contamination as will be discussed later.

Recently, Aminian et al. [14] published a retrospective case series of 4 patients, between 44 and 81 years of age in whom surgical treatment was planned (cholecystectomy, hernia repair, gastric bypass, and cholecystectomy and hysterectomy), who were later found to have COVID-19 infection.

Two patients developed postoperative ARDS and three of the four patients died, one of them before the planned surgery. One patient only developed postoperative fever. Based on this anecdotal evidence, Aminian et al. conclude that patients undergoing elective surgery should be screened for COVID 19, that elective surgery should be deferred in COVID-19 positive patients, and that postoperative fever and pulmonary complications should raise the suspicion of COVID-19 infection.

We recommend that all patients were tested for SARSCOV-2 before any elective or emergency surgical procedure, even if they are asymptomatic.

According to the recently published "Intercollegiate General Surgery Guidance on COVID-19”, all patients requiring surgery should have a computed tomography (CT) scan of the chest [4]. Finding of COVID-19 ribonucleic acid (RNA) in sputum using reverse transcriptase-polymerase chain reaction (RT- PCR) may be used to confirm the diagnosis, but RT-PCR sensitivity is lower than that of CT (60-80\% vs 97\%) [15].

In case of positive findings, elective surgery should be deferred, and in the case of emergent surgery, the risk of increased mortality should be considered and an appropriate consent form signed by the patient.

As far as colorectal cancer care is concerned, we found ourselves facing new problems that have been dealt with in different ways in different regions of Italy according to the capabilities of local health care systems. In the COVID-19 
era, the number of surgical procedures has been reduced everywhere, either because the need for anesthesiologists and beds in intensive-care units (ICUs) for COVID-positive patients has increased enormously, or because the immunosuppression induced by surgery may increase the risk of COVID-19 infection. Based on these considerations and although delays in surgical treatment beyond 2-3 months result in a higher recurrence rate [16], there have been recommendations by societies and experts to delay surgical treatment for stage I and stage II colorectal cancer for up to 6 months [17]. Moreover, neoadjuvant treatments have been recommended for high-risk colon and rectal cancer to defer as long as possible surgical admission [18] with the use of preoperative chemotherapy for colon cancer [17] or of consolidation chemotherapy after either chemoradiation or short-course radiotherapy for rectal cancer $[19,20]$.

However, while temporizing strategies may be useful in case of extreme scarcity of heath care resources or in cases of serious threats to patient safety, the experience which we have matured, especially in northern Italy during the last 2 months, leads us to the following considerations for COVID-19-negative patients with colorectal cancer:

1. We do not know when things will go back to normal. We cannot postpone surgical treatment under the assumption that the risk of hospitalization will be lower in the near future. Moreover, none of the proposed neoadjuvant treatments have been shown to be superior to the more traditional approach and all involve prolonged chemotherapy that will further impair the patient's immune system increasing the risk of COVID-19 complications.

2. Hopefully, elective cancer care can be provided in selected and approved COVID-19-free hospitals. In this ideal situation, all patients should be screened $24-48 \mathrm{~h}$ prior to admission with a chest CT scan and the available rapid turnaround RT-PCR test. All hospital personnel in COVID-19-free hospitals should also be screened by checking their temperature at the hospital entrance and repeated sputum or nasal swab testing, since, for such a system to work, it is necessary to reduce the risk of contamination both from within and from without. Alternatively, if the implementation of COVID-19-free hospitals is unfeasible, elective cancer care surgery may be offered by hospitals where COVID-19-positive and COVID-19-negative patients are located in clearly separate areas which include wards, operating rooms, ICUs, radiology and endoscopy units, and personnel to reduce to a minimum the risk of infection.

3. In general, oncologic protocols should be applied following the current guidelines. Among these protocols and whenever supported by the literature, treatment should be minimized. For locally advanced rectal cancer, several recommendations are available and sup- ported by national and international guidelines. However, within this heterogeneous group of rectal cancer patients, there are subgroups with different risks of recurrence and prognosis ("The Good, the Bad and the Ugly"). Within the context of a pandemic COVID-19 infection, it is reasonable to recommend less aggressive approaches for less aggressive cancers. For example, on approaching rectal cancer determined by clinical staging and magnetic resonance imaging (MRI) to be T3a-b N0 (The Good), upfront total mesorectal excision could be the best treatment as the benefits of chemoradiation therapy followed by total mesorectal excision in this group of patients are questionable. Again, aggressive neoadjuvant therapies with induction or consolidation chemotherapy should be reserved for cancers with the highest risk of recurrence (The Ugly) such as those with infiltration of the mesorectal fascia or clearly positive nodes in the mesorectum or pelvic extramesorectal lymph-node metastasis. Whenever possible, neoadjuvant short-course radiotherapy should be preferred to neoadjuvant chemoradiation therapy and adjuvant chemotherapy should be restricted to the cases where the benefits clearly outweigh the risks, especially elderly patients. Exclusion or delayed surgery should be recommended in those very exceptional situations where anesthesiologists or operating rooms are unavailable, because they are being used to face the pandemic. All these decisions should be made by a multidisciplinary oncology team and fully discussed with patients.

4. As far as the surgical approach for colorectal cancer, the following considerations apply:

Where non-surgical treatment may reach the same goal as surgical therapy, the non-surgical treatment should be preferred. For example: stents should be preferred to palliative resections. When evaluating different surgical options, the rate and severity of postoperative complications should be considered. For example: in patients with low rectal cancer and serious comorbidities with or without prior radiation, Hartmann's procedure may be preferred to the standard reconstruction which is at high risk for anastomotic leak and likely will require a long recovery period and ICU admission. This applies even more to COVID-19 positive patients.

Rectum-sparing approaches (watch and wait) have been suggested as an option in rectal cancer patients with a complete or near-complete clinical response after neoadjuvant therapy. We believe that rectum-sparing approaches are feasible, and oncological outcomes such as overall survival and disease-free survival are likely comparable to those after radical surgery provided that patient selection is optimal. However, as these approaches are not standard of care, it seems reasonable to enter patients into prospective studies where conservative approaches are 
evaluated [21]. Conversely, we discourage rectum-sparing approaches outside of research protocols.

In conclusion, we believe that the COVID-19 epidemic should not lead to approaches that impair oncologic results or expose patients to excessive morbidity. In COVID-19-negative patients, elective surgery should be performed following the current guidelines, using the least aggressive treatment possible and providing treatments in COVID-19-free hospitals (hopefully) or in hospitals where COVID-19-positive and -negative patients follow clearly separate pathways. In COVID-19-positive patients, recovery from the infection is the priority and cancer surgery should be reserved only for life-threatening situations. In both COVID-19-positive and COVID-19-negative cancer patients with an emergent presentation, the treatment should be as conservative as possible, avoiding surgery if feasible, using stent placement for stenosing cancer as bridge to surgery or as palliative treatment. Hartmann's procedure should be considered instead of a low colorectal or coloanal anastomosis, or in presence of left-sided occlusion or perforation. Ostomies should be strongly considered.

Other viruses have shown an increased release during laparoscopy with carbon dioxide. The risk of aerosol exposure and subsequent infection for the surgical team during a minimally invasive procedure is a potential issue [22] which has led scientific societies to recommend performing open surgery in COVID-19-positive patients [4]. However, the potential hazards of laparoscopy (including robotic surgery) need to be weighed against the benefits of a shorter length of stay and decreased complication rate. Laparoscopic surgery in COVID-19-positive patients should be performed in a negative pressure room if available, and an ultra-filtration (smoke evacuation system or filtration) should be used, if available. All pneumoperitoneum should be safely evacuated via a filtration system before closure, trocar removal, specimen extraction, or conversion to open [6]. Moreover, appropriate trocar-size incisions to avoid air leaks are strongly recommended and the use of cautery should be minimized to decrease smoke concentration.

This period of rigorous quarantine is fundamental and necessary to reduce the spread of the virus. Of course, the centralization of treatment of colorectal diseases in some referral centers would be preferable, but common sense suggests that in these months, all the available resources must be directed to the treatment of the COVID19 infection.

Acknowledgements The authors wish to thank Dr D Aiello, A Bondurri, M Fiorino, S Mancini, G Milito, and A Serventi for their advise on the manuscript.

\section{Compliance with ethical standards}

Conflict of interest The authors declare no conflict of interest.

Ethical approval This article does not contain any study with human participants or animals performed by any of the authors.

Informed consent For this type of study, formal consent is not required.

\section{References}

1. Zhou F, Yu T, Du R et al (2020) Clinical course and risk factors for mortality of adult inpatients with COVID-19 in Wuhan, China: a retrospective cohort study. Lancet S0140-6736(20):3056630573 (in press)

2. World Health Organisation (2020) WHO Director General's opening remarks at the media briefing on COVID-19-11 March 2020. WHO, Geneva

3. Spina S, Marrazzo F, Migliari M, Stucchi R, Sforza A, Fumagalli R (2020) The response of Milan's Emergency Medical System to the COVID-19 outbreak in Italy. Lancet 395(10227):e49-e50

4. https://www.acpgbi.org.uk/news/urgent-intercollegiate-generalsurgery-guidance-on-covid-19/

5. https://www.facs.org/about-acs/covid-19

6. https://www.sages.org/recommendations-surgical-response-covid $-19$

7. Lewis R (2020) Early GI Symptoms in COVID-19 may indicate fecal transmission. Gastroenterology. https://doi.org/10.1053/j. gastro.2020.02.054 (in press)

8. Huang C, Wang Y, Li X et al (2020) Clinical features of patients infected with 2019 novel coronavirus in Wuhan, China. Lancet 395:497-506

9. Gu J, Han B, Wang J (2020) COVID-19: Gastrointestinal manifestations and potential fecal-oral transmission. Gastroenterology. https://doi.org/10.1053/j.gastro.2020.02.054 (in press)

10. Zhang J, Wang S, Xue Y (2020) Fecal specimen diagnosis 2019 novel coronavirus-infected pneumonia. J Med Virol. https://doi. org/10.1002/jmv.25742 (in press)

11. Repici A, Maselli R, Colombo M et al (2020) Coronavirus (COVID-19) outbreak: what the department of endoscopy should know. Gastrointest Endosc S0016-5107(20):30245-30255. https ://doi.org/10.1016/j.gie.2020.03.019

12. https://ecco-ibd.eu/images/6_Publication/6_8_Surveys/2nd_Inter view_COVID-19_ECCO_Taskforce_published.pdf

13. https://ecco-ibd.eu/images/6_Publication/6_8_Surveys/3rd_Inter view_COVID-19_ECCO_Taskforce_published.pdf

14. Aminian A, Safari S, Razeghian-Jahromi A, Ghorbani M, Delaney CP (2020) COVID-19 outbreak and surgical practice: unexpected fatality in perioperative period. Ann Surg (in press)

15. Ai T, Yang Z, Hou H et al (2020) Correlation of chest CT and RT-pcr testing in coronavirus disease 2019 (COVID-19) in China: a report of 1014 cases. Radiology. https://doi.org/10.1148/radio 1.2020200642

16. Kucejko RJ, Holleran TJ, Stein DE, Poggio JL (2020) How soon should patients with colon cancer undergo definitive resection? Dis Colon Rectum 63:172-182

17. Seymour MT, Morton D (2019) FOxTROT: an international randomised controlled trial in 1052 patients (pts) evaluating neoadjuvant chemother- apy (NAC) for colon cancer. J Clin Oncol 37:3504-3504. https://doi.org/10.1200/JCO.2019.37.15_suppl .3504

18. https://aischannel.com/colorectal-management-covid-19/ 
19. Marco MR, Zhou L, Patil S et al (2018) Consolidation mFOLFOX6 chemotherapy after chemoradiotherapy improves survival in patients with locally advanced rectal cancer: final results of a multicenter phase II trial. Dis Colon Rectum 61:1146-1155

20. Nilsson PJ, van Etten B, Hospers GA et al (2013) Short-course radiotherapy followed by neo-adjuvant chemotherapy in locally advanced rectal cancer-the RAPIDO trial. BMC Cancer 13:279

21. Barina A, De Paoli A, Delrio P et al (2017) Rectal sparing approach after preoperative radio- and/or chemotherapy $(\mathrm{RESARCH})$ in patients with rectal cancer: a multicentre observational study. Tech Coloproctol 21:633-640
22. Alp E, Bijl D, Bleichrodt RP, Hansson B, Voss A (2006) Surgical smoke and infection control. J Hosp Infect 62:1-5

Publisher's Note Springer Nature remains neutral with regard to jurisdictional claims in published maps and institutional affiliations. 